\title{
Value maximization versus directed exploration in human learning
}

\author{
Ohad Dan ${ }^{1,2}$ and Yonatan Loewenstein ${ }^{1,2,3,4}$
}

\begin{abstract}
${ }^{1}$ Department of Cognitive Sciences, The Hebrew University, Jerusalem, Israel; ${ }^{2}$ The Federmann Center for the Study of Rationality, The Hebrew University, Jerusalem, Israel; ${ }^{3}$ The Alexander Silberman Institute of Life Sciences, The Hebrew University, Jerusalem, Israel; ${ }^{4}$ The Edmond and Lily Safra Center for Brain Sciences, The Hebrew University, Jerusalem, Israel
\end{abstract}

\section{Abstract}

Value maximization and directed exploration are two forces that guide reinforcementlearning agents when facing the exploration-exploitation dilemma. Traditional bandit tasks entangle random and directed exploration thus making it difficult to understand and quantify the role of directed exploration in human learning. Here we introduce a novel experimental framework that allows the isolation of the effects of directed exploration and contrasts it with value maximization. In a 3-armed bandit task, the partial-cyclic feedback reveals the outcomes of the chosen alternative and additional alternative in a specific "informationdirection" such that consecutive choices in the additional alternative complete a choice cycle. Interestingly, both value maximization and directed exploration predict a violation of transitivity, but importantly they predict transitivity would be violated in opposite directions. Value maximization predicts that random fluctuation in value will induce an advantageous perception of the additional alternative, thus rendering information-wise violation of transitivity. In contrast, driven by uncertainty minimization, directed exploration will drive decision-makers to choose the alternative which remained hidden in the previous trial, thus rendering a counter information-wise violation of transitivity. Testing human behavior in this setting, we found that participants violated transitivity in a counter information-wise direction, pointing to the substantial influence directed exploration had on decisions. We discuss the role of directed exploration in human learning and the unintuitive violation of transitivity as an acquired feature resulting from learning.

Keywords: Directed-exploration, Reinforcement-learning, Transitivity, Rationality 


\section{Introduction}

Consider a diner which needs to pick a restaurant in a city with three restaurants arranged in a one-way circle around the city square. To assist the city's diners in their choices, the restaurants advertise their daily menus on large billboards pointed at the direction of the oneway road, but they do so, inconveniently, exactly at noon when lunch is served. As a consequence, if our diner will spend time reviewing the day's menus, she will have no chance of getting a seat in any of the restaurants. Rather, she is forced to choose the restaurant of the day based on her past experiences. Thus, every day while dining she learns how fitting is the restaurant of the day for her taste. However, on a given day her information accumulation is not constrained to the single restaurant she chose, since while she dines she also observes the billboard of the next restaurant (the one in the direction of one-way drive). While she learns how fitting is the "next" restaurant's menu for her, due to the directionality of billboards she cannot learn on that of the "previous" (Fig. 1). What patterns of behavior should be expected in this setting? Naively, the diner is expected to gradually learn which of the restaurants is "best" for her and then, for the majority of her lunches continue visiting it. Specifically, in the context of consistent preferences, a naïve expectation would be that the diner will maintain transitive preference over the restaurants. However, surprisingly, this must not be the case. We show here that utilizing this unique type of feedback, both theoretical models of learning predict, and human behavior demonstrates a systematic violation of transitivity.

Preferences-transitivity is a fundamental principle of the rational perspective (Morgenstern \& Von Neumann, 1953; Savage, 1972). Preferences-transitivity over a set of alternatives implies that every three alternatives may be consistently ordered according to their preference. Namely, given three alternatives $a_{1}, a_{2}, a_{3}$ if $a_{1} \geqslant a_{2}$ and $a_{2} \geqslant a_{3}$ a transitive relation implies that $a_{1} \geqslant a_{3}$ ( $\geqslant$ symbolizing weak preference, i.e. preference or indifference). To illustrate this, consider again the diner which is provided with menus of the three restaurants and is asked to indicate which one of every two menus she prefers. It would be a violation of transitivity, and indeed very strange, if the diner will report preferring $a_{1}$ over $a_{2}, a_{2}$ over $a_{3}$ but also $a_{3}$ over $a_{1}$. With well-studied exceptions (see for example, BarHillel \& Margalit, 1988; Brandstätter et al., 2006; Gonzalez-Vallejo, 2002; Tversky, 1969), humans mostly maintain transitive preferences over a given set of choices (Regenwetter et 
al., 2011). Moreover, when confronted with an inconsistent preference they expressed, humans consider it a mistake (Luce \& Raiffa, 1989).

Transitivity violation in repeated choices is not trivial to demonstrate (Regenwetter et al., 2011). For example, a transitive preference may be formulated probabilistically (a condition sometimes termed weak stochastic preference, Block et al., 1959; Suppes, 1965; Tversky, 1969). Then, a most preferred alternative implies being associated with the highest choice probability (Davis, 1958). Even for these conditions, one particular way to violate transitivity is a case in which the most preferred alternative is conditioned on a previous choice. For example, an agent that most prefers alternative $a_{3}$ if $a_{1}$ was previously chosen, but also holds $a_{2}$ as its most preferred alternative if $a_{3}$ was just chosen. In this scenario $a_{2}$ and $a_{3}$ are both, sometimes (conditioned on the previous choice), most preferred in a way that does not allow to order preferences, thus violating transitivity. To test whether humans violate transitivity using this criterion, we utilized a novel type of feedback.

In the context of learning from experience, feedback is the mechanism by which the outcomes of previous choices are reported to decision-makers. The most prevalent types of feedback are the full and partial feedback in which the single outcome of the chosen alternative or outcomes of all alternatives respectively are revealed to the decision-maker following a choice. Here, we introduce a novel type of feedback, an intermediate version of the partial and full feedback we term the partial-cyclic feedback. Similar to the learning of the diner presented in the first paragraph, the partial-cyclic feedback is implemented in a 3-armed bandit setting (Bergemann \& Valimaki, 2006) in which decision-makers learn on the outcome of their single choice but also on the outcome of additional unchosen alternative in a specific "information direction" such that consecutive choices in that additional alternative yield a cyclic choice pattern over the alternatives (Fig. 1). More explicitly, given three alternatives $\left\{a_{1}, a_{2}, a_{3}\right\}$, a choice in $a_{1}$ yields the outcome of $a_{1}$ only, but reveals the outcomes of alternatives $a_{1}$ and $a_{2}$, however not $a_{3}$; similarly, a choice in $a_{2}$ reveals the outcomes of $a_{2}$ and $a_{3} ;$ and a choice in $a_{3}$ reveals the outcomes of $a_{3}$ and $a_{1}$. Here we explore the consequences of utilizing the partial-cyclic feedback on participants' preference-transitivity. Specifically, we ask whether the partial-cyclic feedback affects decision-makers' choices such that they will violate transitivity. To answer this question we first ask, what are the forces which guide decision-makers' choices in such an environment? 
An obvious candidate to consider when assessing the forces that shape decision-making in decisions from experience is value maximization. In a most trivial manner, biological entities aim to improve their prosperity by maximizing present and future prospects. In a context of learning, the algorithms utilized for such optimization are extensively studied both empirically in the field of operant conditioning (Mongillo et al., 2014; Shteingart \& Loewenstein, 2014) and theoretically in the field of reinforcement learning (RL, Sutton \& Barto, 2018). One particular algorithm that is widely used both to model biological decision making and in theoretical applications is Q-learning (Sutton \& Barto, 2018; Watkins \& Dayan, 1992). Briefly, Q-learning associates a "Q-value" with actions, a value that represents the action's expected average reward. To optimize prospects, decision-makers utilize these learned Q-values to choose between actions by assigning greater choice probabilities to actions with greater Qvalues (Methods). We argue and then show that applying the Q-learning algorithm utilizing the partial-cyclic feedback may induce unintuitive patterns of behavior.

Consider three alternatives that are associated with identical stochastic reward distributions that yield either "high" or "low" values. Given some preference the agent presently has over the three alternatives, it is more likely to choose the alternative it currently considers as best. The feedback provided to the agent following its choice may reinforce the current evaluation of the goodness of the best alternative. However, by random fluctuations of the values drawn from the alternatives' reward distributions, the value presented in the information-direction may change the perception of the additional alternative so as to be considered as the best alternative, thus increasing the probability of choice in the information direction. Repeating this process, simply as a function of value update (namely, learning), the agent is constantly biased to switch its current preference in one specific direction. Thus, value maximization is expected to drive decision-makers to violate transitivity in the information direction.

A less obvious force that may drive decisions is directed exploration (Fox et al., 2020). In an attempt to maximize their prospects, utilizing any feedback but the full-feedback, decisionmakers are faced by the exploration-exploitation dilemma (Gershman, 2019; Mehlhorn et al., 2015; Sutton \& Barto, 2018). If they only choose the currently seeming best alternative, decision-makers will remain less certain about the potential outcomes of other alternatives that could potentially hold even greater rewards. Thus, decision-makers are faced with two often opposing choices, choosing better alternatives on the one hand, exploitation, or 
reducing uncertainty on the other hand, exploration. In such cases that decision-makers choose to explore, several methods exist for making an exploratory choice, one of which is directed exploration (see Schulz \& Gershman, 2019). According to this principle, decisions are directed to actions that are associated with greater uncertainty. How will directed exploration be manifested when utilizing the partial-cyclic feedback? Following a choice, decision-makers are informed on the outcomes of their chosen alternative and additional alternative in the information-direction, while the outcome of the third alternative, the one in the counter information direction, remains hidden. In this case, the recent relative uncertainty is increased in that third alternative which remained hidden. Thus, directed exploration prescribes a higher choice probability to the alternative in the counter information direction. Repeating this process, choices will be constantly biased in the counter information direction, rendering an intransitive preference over the three alternatives in the opposite direction of the information direction.

Taken together, value maximization and directed exploration each suggests that in the partialcyclic feedback environment decision-makers should violate transitivity but, interestingly, predict that this violation would be in opposite directions. Using simulations, we specify the predictions of theoretical learning models and then using behavioral experiments test whether humans do in fact violate transitivity and if so in which direction. Applying this behavioral paradigm, we test the role of value maximization and directed exploration in human decision making and their relative influences on behavior.

\section{Reward $=r_{\mathrm{A}}$}
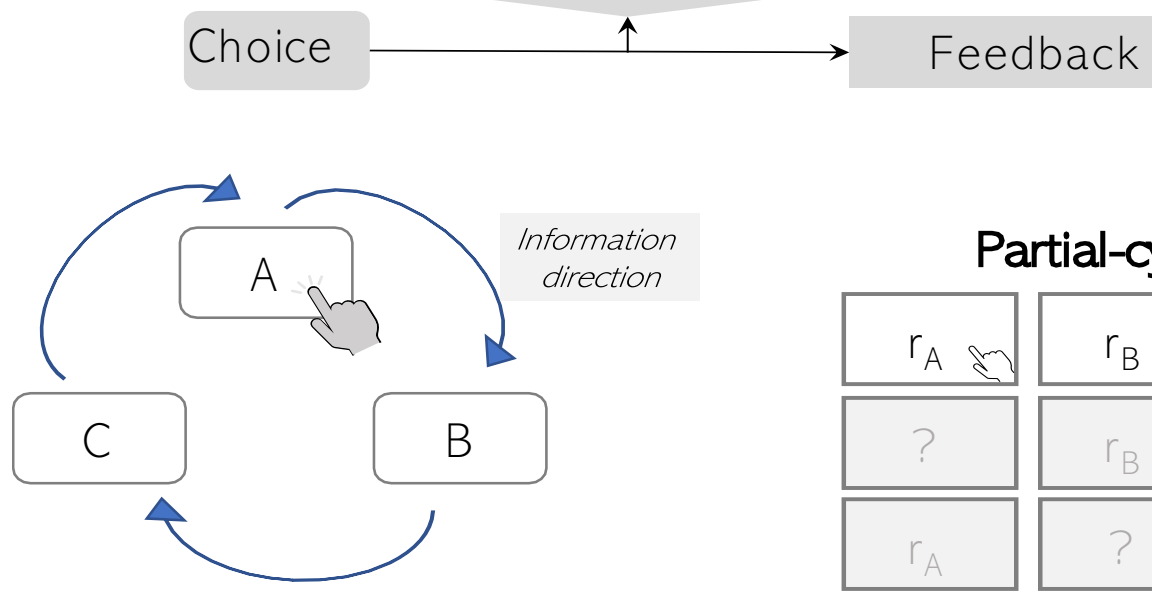

Partial-cyclic

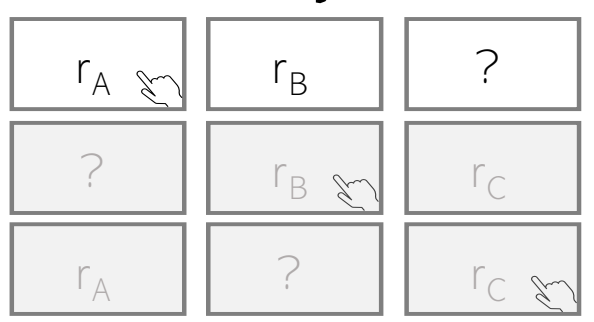


Fig. 1. Partial-cyclic feedback. In a 3-armed bandit task, a choice in alternative $A$ (left) yields a single reward $r_{A}$ but reveals both $r_{A}$ and the reward in additional alternative located in the "information direction" (direction of blue arrows), $r_{B}$. Note the reward in the "anti-information direction", $r_{c}$, remains hidden (White rectangles). Similarly, choices in $B$ reveals $r_{B}$ and $r_{C}$, and choice in $C$ reveals $r_{C}$ and $r_{A}$ (grey rectangles).

\section{Results}

To test what behavior is expected under the partial-cyclic feedback regime, we first simulated learning using a Q-learning model (Methods). In 100 repetitions, we simulated agents' decision making under the partial-cyclic feedback in repeated choices. As entailed by the partial-cyclic feedback, a choice in one of the alternatives revealed the reward granted by the chosen alternative and an additional forgone reward of one other alternative (Fig. 1, Methods). The rewards were drawn from a bi-modal normal distribution, constructed such that in each draw there was a 50\% chance of drawing a "high" reward and a 50\% chance of a "low" reward (Methods). Observing overall behavior, we tested whether the simulations demonstrated a transitive preference over the three alternatives. Specifically, we contrasted the probability of information-wise transitions in consecutive trials (choice in the additionally presented alternative, $B$ in Fig. 1), with the probability of counter information-wise transitions (the alternative which remains hidden, $C$ in Fig. 1). We found that the simulated agents expressed a preference for information-wise transitions compared to counter information ones and that the magnitude of this preference depended on the agent's learning rate (Fig. 2.a). For example, for a learning-rate value $\eta=0.4,26 \%$ of the transitions between subsequent trials were information-wise, significantly more than counter information-wise transitions which constituted only $13 \%$ of the transitions between subsequent trials (2-tailed t-test, $p<0.001$, Fig. $2 . b$; in the rest of the transitions agents employed a "stay" strategy and repeatedly chose the same alternative in subsequent trials). The extent of this preference crucially depended on the agents' learning rate parameter, such that greater learning rate led to a greater preference for information-wise transitions (Fig. 2.a). 
To test if human participants violate transitivity in a similar way, we conducted a behavioral experiment in which 66 participants repeatedly chose between three alternatives utilizing the partial-cyclic feedback, while being incentivized to maximize their per-trial reward (see Methods). Importantly, to minimize the chance of confounding our results with participants' a priori preference (or anti preference) to clock-wise transitions we randomly determined the "information direction" between participants (direction of blue arrows in Fig. 1; for each participant the chosen direction was constant across the experiment). Specifically, participants were randomly assigned to either a clockwise-information condition or counter clockwise-information condition (choice in $A$ in Fig. 1 also revealed either the reward of $B$ or $C$, respectively). To be able to collapse the two conditions into a single analysis, we verified that participants' transition probability in sequential trials did not significantly differ between the two condition (2-tailed $t$-test): information-wise $(t=0.43, p=.67, d f=64)$, counterinformation-wise ( $t=1.70, p=.09, d f=64)$ and no transition in subsequent trials ("stay", $t=1.62$, $p=.11, d f=64)$.

Concluding that the actual direction of information presentation did not play a major role in determining participants' preferences, we then collapsed the two conditions and simply considered information-wise and counter information-wise transitions. We found that participants indeed violated transitivity by expressing a significant and substantial preference towards one type of transitions over the other. However, opposite the results of the value-maximizing agents in simulations, participants robustly preferred counter information-wise transitions over information-wise ones (choice in $C$ rather than $B$ in Fig. 1). Participants' overall proportion of counter information-wise transitions was $39 \%$, significantly more than the proportion of information-wise transitions, 25\% (2-tailed t-test, $t=3.57 p<0.001, d f=65$, Fig. 2.c). 
a.

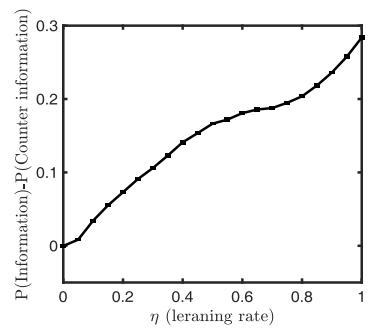

b.
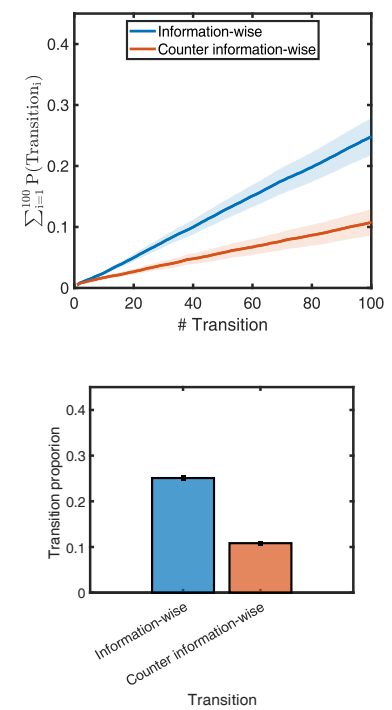

c.
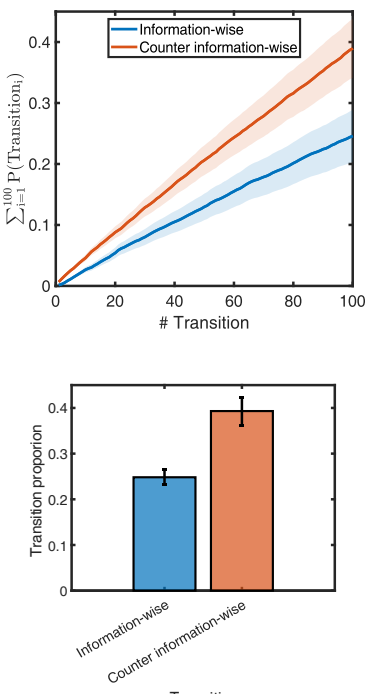

Fig. 2. Transitivity violation in the partial-cyclic feedback. a. Simulation - Informationwise preference increases with learning rate. The average relative preference of information-wise transitions (a choice in $A$ and then $B$ in Fig. 1) over counter information-wise transitions (a choice in $A$ and then $C$ in Fig. 1 ) in subsequent trials is evaluated for different learning rates using a simple reinforcement learning model (0 on the ordinate representing an equal preference for the two transition types). Error bars are standard error of the mean calculated over 100 repetitions per each learning rate. As shown by the figure, the greater the learning rate the greater is the learninginduced bias for information-wise transitions. b. Simulation - Information-wise preference for a specific learning rate, along trials. Per each transition (top panel, abscissa) using a simple reinforcement learning model with a specific learning rate, $\eta=0.4$ (i.e. a single data point from Fig. 3.a) the probability of each transition type is evaluated. The ordinate of the top panel is the cumulative sum of information-wise and counter information-wise transition probabilities (blue and red lines respectively, shaded area is standard error of the mean calculated over 100 simulations). Note that an additional transition type, "stay", where the same alternative is repeatedly chosen in subsequent trials, is not plotted. The bottom panel quantifies the overall proportion of information-wise and counter information-wise transitions (error bars are standard error of the mean calculated over the individual proportions for each of the 100 
independent simulations). C. Preference for counter information-wise transitions in behavior. Top and bottom panels display data from 66 participants in the same format as b. Opposite the simulation results, human participants demonstrate a robust preference for counter information-wise transition, i.e. preferring to choose the alternative whose outcome remained hidden in the previous trial.

To further explore the preference for counter-information transitions demonstrated in the population level, using maximum-likelihood estimation we fit the behavior of individual participants to a model that combines the forces of value maximization and directed exploration. For a complete description of the model see Methods. Briefly, the model assigns a choice probability $P\left(a_{i}\right)$ to an action $a_{i}$, a probability that is the weighted sum of two forces. With a weight of $\frac{\alpha}{2}(-1 \leq \alpha \leq 1)$, the model exclusively prefers $a_{i}$ if it is the target of directed exploration, namely if it is the counter-information alternative relative to the previous choice ( $C$ in Fig. 1). With a complementary weight $1-\frac{\alpha}{2}$, the model assigns a choice probability to $a_{i}$ by considering the estimated value of $a_{i}, Q_{a_{i}}$, relative to the values of all alternatives, $Q_{A}$, such that actions with greater values are prescribed higher probabilities. The differential preference to better alternatives is determined by the softmax function, which itself parameterizes the sensitivity to value differences by a dedicated parameter ( $\beta$, see Methods):

Eq. $1 \quad P\left(a_{i}\right)=\frac{\alpha}{2}($ directed exploration $)+\left(1-\frac{\alpha}{2}\right) * \operatorname{softmax}\left(Q_{a_{i}}, Q_{A}\right)$

Fitting behavior to this model, great values of $\alpha$ (close to 1 ) imply that decision making is largely driven by directed exploration; small $\alpha$ values (close to -1 ) imply that decision-makers are "aversive" to directed exploration choices (prefer stay or information-wise transitions); and, $\alpha$ values close to 0 imply that the agent is only marginally influenced by directed exploration and is mainly driven by value maximization (as prescribed by the Q-learning algorithm). We find variability in the values of $\alpha$ fitted to participants (Fig. 3). However, overall the $\alpha$ distribution is significantly biased towards positive $\alpha$ values compared to the $\alpha$ values fitted to simulations whose $\alpha$ are fixed on 0 ( $p<0.001$, see Methods, Fig. 3). The mean value of $\alpha$ is 0.25 , with $86 \%$ of participant having positive $\alpha$ values. 


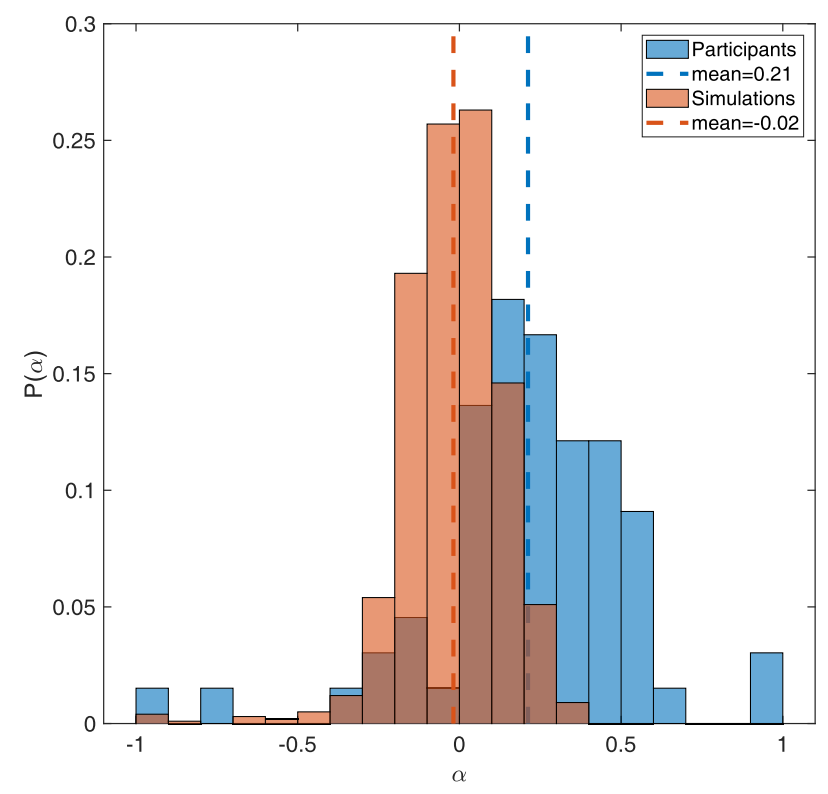

Fig. 3. Directed exploration parameter $(\alpha)$ for participants and simulations. A model that combines value maximization and directed exploration (Eq. 1) is fitted to individual participants' behavior. Blue bars are histogram of participants' extracted $\alpha$, and red bars are histogram of the distribution of $\alpha$ values fitted to behavior assuming the participants' mean rate $\eta=0.5$. Dashed line is the distributions' means. As portrayed by the figure, the two distributions are largely distinct, with the majority of participants' $\alpha$ s being greater than those in simulations. Note that the maximum-likelihood estimator used to fit the model is biased, i.e. the mean distribution is slightly $(-0.02)$ but significantly $(p<0.001)$ different than 0 , although the real $\alpha$ used in simulations is 0 (see Discussion).

\section{Discussion}

Preference-transitivity is commonly observed in human behavior. Moreover, preferencetransitivity is axiomatically required for an agent to deploy rational behavior, and deviations from it are interpreted as mistakes. Naively surprising, here we show that in a setting of learning from experience humans violate transitivity. Using simple computational models, we show how this pattern is an expected outcome of learning. Within the theoretical framework we introduce, we point out two factors that can cause a violation of transitivity. In the 3armed bandit task and partial-cyclic feedback we utilized, the first force, value maximization, should drive behavior in the information direction. Opposite to it, directed exploration should drive behavior in a counter information direction. In a behavioral experiment, we show that 
participants indeed violate transitivity and that this violation is overall in a counter information direction. Fitting the behavior of individual participants to a model that parameterizes the relative forces of value maximization and directed exploration, we find heterogeneity in the population which is characterized by a pronounced bias towards a positive preference for directed-exploration.

Surprisingly, given naïve expectations, our participants robustly violated transitivity. This, however, does not mean we now expect to encounter more and more people that systematically violate transitivity. While some studies do identify decision-makers' violation of transitivity (e.g. Diaye \& Urdanivia, 2009; Loomes et al., 1991) when tested in real-world scenarios, humans are not likely to be vulnerable to methodical exploitation of such irrationalities, e.g. if subjected to the notorious "money-pump" (Echenique et al., 2011; Voorhoeve \& Binmore, 2006). Furthermore, when confronted with an instance of transitivity violation people wish to "fix" their preferences to comply with transitivity (Luce \& Raiffa, 1989).

One, not completely technical, issue with our results is the parameter fitting procedure. When fitting simulated behavior to our model (Eq. 1) using maximum-likelihood, we find that the resulting estimator of $\alpha$ is systematically negatively biased. Namely, that even when the behavior we fit utilizes an $\alpha$ value which we fix at 0 , the mean $\alpha$ we reconstruct is negative. One way to conceptualize this bias is that preference for transitions of type stay and information-wise are, on average, consistent with an "aversion" to directed exploration, and differentiating between the two is difficult for the outside observer. This is in fact one of our prime theoretical predictions. In the experimental framework we introduce, we predict that a model with no explicit attitude towards directed exploration will still violate transitivity in the information direction, namely, behave as if it is aversive to choices in the hidden alternative. Thus, the negative bias in $\alpha$ estimation and the long negative tail in the $\alpha$ distribution (see Fig. 3) are expected outcomes of fitting the behavior in our environment. In light of this, the significant positive average $\alpha$ we identify in behavior even more robustly emphasizes the role of directed exploration in human behavior as it is likely an underestimation of the "true" $\alpha$ value. 
What is the allure of the hidden information empirically demonstrated by our participants? While the cyclic-preference our simulated-agents demonstrated was information-wise, participants' preferences were counter information-wise. If we assume that participants are value-maximizers, then they are by definition more likely to choose alternatives that they perceive as more valuable. This would imply that in consecutive trials,participants perceived the hidden alternative (the single alternative whose value is not revealed following a choice; $C$ in Fig. 1) as superior. In terms of standard theories of learning which do not consider directed exploration as an elementary component, such behavior is intriguing. Most theories of learning assume that in the process of learning, the value of unsampled alternatives either does not change or undergoes a regression to a mean value (e.g. Plonsky \& Erev, 2017; see Sutton \& Barto, 1998). It is hence not trivial to accommodate the preference for an alternative which likely was less valued in the previous trial (given that it was not chosen) within such models.

Several approaches may explain the preference for the hidden alternative. In the reinforcement-learning perspective on exploration (Sutton \& Barto, 2018; Thrun, 1992), directed exploration describes the assignment of an added value to actions that are associated with greater uncertainty, e.g. in the form of exploration bonus (for a review, see Gershman, 2018). Previous studies have found that humans indeed utilize directed exploration when they face the exploration-exploitation dilemma (Gershman, 2019; Krebs et al., 2009; Wilson et al., 2014). In this view, the hidden alternative in our experiment is associated with greater, though often just recent, uncertainty. Utilizing directed exploration, the next choice will be directed to reduce this uncertainty, hence the hidden alternative will be more likely chosen. A related approach, also from the perspective of reinforcement learning, is to consider the preference for the hidden alternative as an extreme form of "optimism in the face of uncertainty" (Szita \& Lőrincz, 2008). Similar to exploration bonus, such optimism is an effective exploration strategy which ascribes higher values to states with greater uncertainty. However, optimism is often used as a means for value initialization (see for example Shteingart et al., 2013) and the application of the optimism principal to increase the value of unobserved alternatives repeatedly in multiple trials is unconventional. A different interpretation of the preference for hidden information is to explain it as driven by curiosity. Both computational (Burda et al., 2018; Savinov et al., 2018) and behavioral theories 
(Berlyne, 1978; Collins et al., 2004) characterize curiosity, the intrinsic motivation to know, as a powerful force. However, to consider curiosity towards an alternative that was just chosen (in a previous "cycle" of the cyclic preference) is, at best, a peculiar application of the term. Yet another, less quantitative but maybe more suitable characterization of our results is the psychological phenomenon known as the fear-of-missing-out, according to which "the party is always somewhere else" (Przybylski et al., 2013). Such exploration heuristic implies that unchosen alternatives would seem more attractive due to the possibility (the "fear") that "missing" the prospects of the hidden alternative is more painful than letting go of alternatives whose values are presently known. Altogether, a learning algorithm that does not integrate some form of directed-exploration as an elementary component would seem insufficient to explain our results.

In conclusion, our findings contribute to recent literature which highlights the importance of directed exploration to human learning and decision making (Fox et al., 2020). Future studies may address the preference for hidden information directly to pinpoint the behavioral mechanisms giving rise to its systematic preference. From an experimental point of view, we introduce a novel type of feedback in the bandit task and show the unintuitive consequences of utilizing it. Finally, in observing a violation of transitivity but also predicting it from theory, we demonstrate how very surprising behavioral outcomes may be trivially explained as an outcome of learning.

\section{Methods}

In the behavioral experiment, participants sequentially chose between three alternatives, choices that were followed by condition-specific information. Participants were randomly assigned to either clockwise or counter-clockwise conditions which dictated the information direction (the direction of blue arrows in Fig. 1). The nature of the feedback utilized in participants' assigned condition was explained to participants without contrasting it with the other type of feedback. Participants were instructed to maximize their per-trial reward and were incentivized to do so by choosing the outcome of one random trial at the end of the experiment as an additional bonus payment. We included in the analysis only and all of the participants who completed their assigned task, choices in 101 trials (to allow for 100 transitions). 
The experiments were run on the online MTurk platform. In all experiments, following the instructions, a screen with several alternatives represented by buttons was displayed. A choice, clicking one of the buttons, disabled all buttons for 1.5 seconds and revealed condition-specific information. To equalize the motoric cost of choices across all the alternatives, progressing to the next trial required pressing a reset button located equidistantly relative to all the alternatives.

The rewards revealed to participants, for any of the alternatives, were drawn from a bi-modal reward distribution:

$$
\text { All alternatives }:=\left\{\begin{array}{l}
\mathrm{P}(r \sim[N(250,60)])=0.5 \\
\mathrm{P}(r \sim[N(750,60)])=0.5
\end{array}\right.
$$

Denoting the alternatives by $a_{1}, a_{2}$, and $a_{3}$, in the clockwise condition a choice in $a_{1}$ also revealed the reward of $a_{2}$, choice in $a_{2}$ also reveled $a_{3}$ and choice in $a_{3}$ also revealed $a_{1}$. In the counter-clockwise condition, the information direction was opposite.

To simulate behavior we utilized a Q-learning model (Sutton \& Barto, 2018), a reinforcement learning algorithm that has been widely used to model sequential decision-making behavior in humans and animals (Barto et al., 1991; Mongillo et al., 2014). Applied to our task, Qlearning describes how a Q-value associated with each action, its expected average reward, is updated given new observations. The initial value of an action is set by the first observation it yields and, inspired by experimental findings (Shteingart et al., 2013), the agent starts its sequence of choices by acquiring an observation for each alternative in random order (a form of optimism, Szita \& Lőrincz, 2008). Following initialization, at each subsequent trial $t$, upon its choice, the agent is informed on the trial's outcome. Namely, some rewards $r_{t}(a)$ associated with actions $a$ are revealed and the agent utilizes this information to update the Q-values of $a$. The updated value $Q_{t+1}(a)$ of a particular action $a$, is a weighted average of the previous value $Q_{t}(a)$ and the received reward:

$$
Q_{t+1}(a)=Q_{t}(a)+\eta\left(r_{t}(a)-Q_{t}(a)\right)
$$

where $0<\eta \leq 1$ is the learning rate. The values of actions that did not receive updated information in a trial remain unchanged. Given the alternatives' present values, the agent chooses one of the actions using an action-selection rule. We utilize a softmax action- 
selection rule, which assigns a choice probability $\left(\operatorname{Pr}\left[a_{i}\right]\right)$ for an action $i$ given all the actions' $(A)$ values:

$$
\operatorname{Pr}\left[a_{i}\right]=\frac{e^{\beta Q_{t}\left(a_{i}\right)}}{\sum_{a \in A} e^{\beta Q_{t}(a)}}
$$

Where $\beta>0$ is an exploration parameter. Where not otherwise specified, we utilized in simulations the parameter values $\eta=0.4, \beta=52$, as extracted from behavior in similar experimental conditions by Shteingart et al., 2013.

To model the relative contribution of directed exploration to decision making we introduced the model of Eq. 1:

$$
\begin{gathered}
P\left(a_{i_{t}}\right)=\alpha(\text { is explore })+(1-\alpha) * \operatorname{softmax}\left(a_{i}, A\right) \\
=\alpha \delta_{a_{t-1}, a_{i}-1}+(1-\alpha) * \frac{e^{\beta q_{i}}}{\sum_{k=1}^{3} e^{\beta q_{k}}}
\end{gathered}
$$

Where $-1 \leq \alpha \leq 1$ quantifies the contribution of directed exploration to a choice, $\delta_{a_{t-1}, a_{i}-1}$ is Kronecker delta that is assigned the value 1 if current transition is of type counter information and 0 otherwise, and $\beta$ is the same exploration parameter presented above. 


\section{References}

Bar-Hillel, M., \& Margalit, A. (1988). How vicious are cycles of intransitive choice? Theory and Decision, 24(2), 119-145.

Barto, A. G., Sutton, R. S., \& Watkins, C. J. C. H. (1991). Learning and Sequential Decision Making. In Learning and Computational Neuroscience (pp. 539-602).

Bergemann, D., \& Valimaki, J. (2006). Bandit problems.

Berlyne, D. E. (1978). Curiosity and learning. Motivation and Emotion, 2(2), 97-175.

Block, H. D., Marschak, J., \& others. (1959). Random orderings and stochastic theories of response.

Brandstätter, E., Gigerenzer, G., \& Hertwig, R. (2006). The priority heuristic: Making choices without trade-offs. Psychological Review, 113(2), 409-432.

Burda, Y., Edwards, H., Pathak, D., Storkey, A., Darrell, T., \& Efros, A. A. (2018). Large-Scale Study of Curiosity-Driven Learning.

Collins, R. P. P., Litman, J. A. A., \& Spielberger, C. D. D. (2004). The measurement of perceptual curiosity. Personality and Individual Differences, 36(5), 1127-1141.

Davis, J. M. (1958). The transitivity of preferences. Behavioral Science, 3(1), 26-33.

Diaye, M. A., \& Urdanivia, M. W. (2009). Violation of the transitivity axiom may explain why, in empirical studies, a significant number of subjects violate GARP. Journal of Mathematical Psychology, 53(6), 586-592.

Echenique, F., Lee, S., \& Shum, M. (2011). The money pump as a measure of revealed preference violations. Journal of Political Economy, 119(6), 1201-1223.

Fox, L., Dan, O., Elber, L., \& Loewenstein, Y. (2020). Exploration: from machines to humans. Current Opinion in Behavioral Sciences.

Gershman, S. J. (2018). Deconstructing the human algorithms for exploration. Cognition, 173, 34-42.

Gershman, S. J. (2019). Uncertainty and exploration. Decision, 6(3), 277-286. 
Gonzalez-Vallejo, C. (2002). Making trade-offs: A probabilistic and context-sensitive model of choice behavior. Psychological Review, 109(1), 137.

Krebs, R. M., Schott, B. H., Schütze, H., \& Düzel, E. (2009). The novelty exploration bonus and its attentional modulation. Neuropsychologia, 47(11), 2272-2281.

Loomes, G., Starmer, C., \& Sugden, R. (1991). Observing Violations of Transitivity by Experimental Methods. Econometrica, 59(2), 425.

Luce, R. D., \& Raiffa, H. (1989). Games and decisions: Introduction and critical survey. Courier Corporation.

Mehlhorn, K., Newell, B. R., Todd, P. M., Lee, M. D., Morgan, K., Braithwaite, V. A., Hausmann, D., Fiedler, K., \& Gonzalez, C. (2015). Unpacking the exploration-exploitation tradeoff: A synthesis of human and animal literatures. Decision, 2(3), 191-215.

Mongillo, G., Shteingart, H., \& Loewenstein, Y. (2014). The Misbehavior of Reinforcement Learning. Proceedings of the IEEE, 102(4), 528-541.

Morgenstern, O., \& Von Neumann, J. (1953). Theory of games and economic behavior. Princeton university press.

Plonsky, O., \& Erev, I. (2017). Learning in settings with partial feedback and the wavy recency effect of rare events. Cognitive Psychology, 93, 18-43.

Przybylski, A. K., Murayama, K., Dehaan, C. R., \& Gladwell, V. (2013). Motivational, emotional, and behavioral correlates of fear of missing out. Computers in Human Behavior, 29(4), 1841-1848.

Regenwetter, M., Dana, J., \& Davis-Stober, C. P. (2011). Transitivity of Preferences. Psychological Review, 118(1), 42-56.

Savage, L. J. (1972). The foundations of statistics. Courier Corporation.

Savinov, N., Raichuk, A., Marinier, R., Vincent, D., Pollefeys, M., Lillicrap, T., \& Gelly, S. (2018). Episodic Curiosity through Reachability. 7th International Conference on Learning Representations, ICLR 2019.

Schulz, E., \& Gershman, S. J. (2019). The algorithmic architecture of exploration in the human 
brain. In Current Opinion in Neurobiology (Vol. 55, pp. 7-14). Elsevier Ltd.

Shteingart, H., \& Loewenstein, Y. (2014). Reinforcement learning and human behavior. In Current Opinion in Neurobiology (Vol. 25, pp. 93-98).

Shteingart, H., Neiman, T., \& Loewenstein, Y. (2013). The role of first impression in operant learning. Journal of Experimental Psychology: General, 142(2), 476-488.

Suppes, P. (1965). Preference, Utility and Subjective Probability. InHandbook of Mathematical Psychology, ed. RD Luce, RR Bush and EH Galanter, 3, 249--410. New York: Wiley.

Sutton, R. S., \& Barto, A. G. (2018). Reinforcement learning: An introduction. MIT press.

Szita, I., \& Lőrincz, A. (2008a). The many faces of optimism: a unifying approach. Proceedings of the 25th International Conference on Machine Learning, 1048-1055.

Szita, I., \& Lörincz, A. (2008b). The many faces of optimism. Proceedings of the 25th International Conference on Machine Learning - ICML'08, 1048-1055.

Thrun, S. B. (1992). Efficient exploration in reinforcement learning.

Tversky, A. (1969). Intransitivity of preferences. Psychological Review, 76(1), 31-48.

Voorhoeve, A., \& Binmore, K. (2006). Transitivity, the sorites paradox, and similarity-based decision-making. Erkenntnis, 64(1), 101-114.

Watkins, C. J. C. H., \& Dayan, P. (1992). Q-learning. Machine Learning, 8(3-4), 279-292.

Wilson, R. C., Geana, A., White, J. M., Ludvig, E. A., \& Cohen, J. D. (2014). Humans use directed and random exploration to solve the explore-exploit dilemma. Journal of Experimental Psychology: General, 143(6), 2074-2081. 